\title{
NUMERICAL SOLUTION FOR THE PARAMETER ESTIMATION OF THE MOISTURE TRANSFER COEFFICIENT
}

\author{
YONG HUN LEE
}

\begin{abstract}
We investigate the estimation of the moisture transfer coefficients in porous media by optimization technique which minimizes the functional defined by the squares error of the numerical solution of an inverse diffusion problem from their experimental values of the moisture content at the some time-steps. In this paper, we solve a diffusion equation numerically by the control volume finite element methods.
\end{abstract}

\section{Introduction}

We consider an nonlinear diffusion equation defined by

$$
\frac{\partial m}{\partial t}=\frac{\partial}{\partial x}\left[D(m, t) \frac{\partial m}{\partial x}\right], \quad t>0, \quad 0<x<L
$$

where $m$ is the moisture concentration, $D(m, t)$ is the coefficient of the moisture transfer, $t$ and $x$ denote time and space coordinate, respectively. In order to make an estimate the moisture transfer in the porous media, we solve the nonlinear diffusion equation (1.1) with some various types of the transfer coefficients. There were many efforts to develop more accurate governing equation and coefficients during recent decades. However, it is very difficult to determine the transfer coefficient which is fulfilled for the various porous material such as brick, soil, concrete and wood. In particular, transfer coefficient of wood is more difficult than other material, because it has the anisotropic and heterogeneous characteristic.

Received January 26, 2010. Accepted April 20, 2010.

2000 Mathematics Subject Classification: 76S05, 65C20; 80A20, 74 G15.

Key words and phrases: parameter estimation, moisture transfer coefficient, control volume finite element method, Gauss-Newton method.

This work was supported by the National Research Foundation of Korea(NRF) grant funded by the Korea government(MEST) (No. 2009-0075067). 


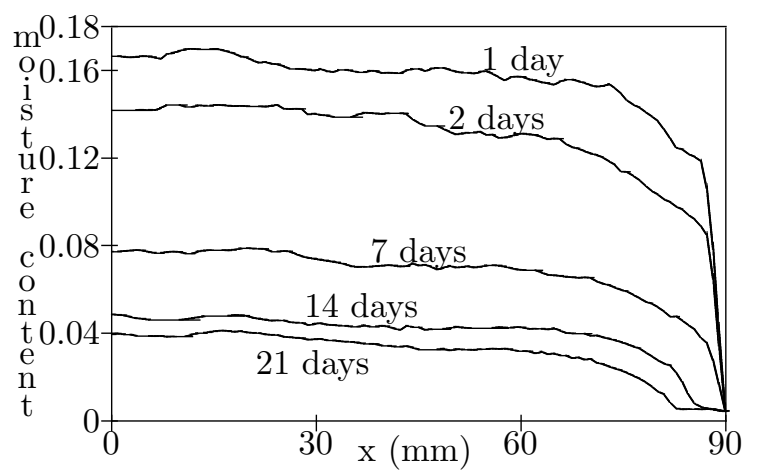

Figure 1.1. The profile of the moisture distribution along the width $x$ of the sample after $1,2,7,14$ and 21 days

In this paper, we investigate the method to find individual transfer coefficients for the given material instead of more accurate form of the general coefficients. For the purpose of it, we define the coefficient $D(m, t)$ in the equation (1.1) as a sum of the exponential form and power form as following:

$$
D(m, t)=p_{1} m^{p(t)}+A e^{-\mu\left(m-\nu_{0}\right)}, \quad p(t)=p_{2}+p_{3} e^{-p_{4} t} .
$$

Then we estimate the parameters by comparing the experimental measured data with the solution of the nonlinear diffusion equation (1.1) with some initial and boundary conditions. So, the experimental quantitative measurements of the moisture space distribution during different time moments are necessary.

The experimental data of the moisture distribution in a rectangular porous sample of size $3 \times 9 \times 12 \mathrm{~cm}^{3}$ during the drying of the sample are obtained in [6]. The measured profiles of [6] are shown in Figure 1.1. All sided surface are water and air-proofed beside one side with dimensions $3 \times 12 \mathrm{~cm}^{2}$ where evaporation of moisture takes place into atmosphere. The moisture transfer coefficient is calculated under the assumption of a one-dimensional moisture movement along the sample's width of the $9 \mathrm{~cm}$ size and is empirically determined in the form of (1.2). the coefficients in (1.2) are calculated under a condition of the minimum of a quadratic functional that determines a diversion of theoretical results obtained by a numerical solution of the nonlinear diffusion equation from the experimental data. 
In this paper, in order to obtain the numerical solution of the nonlinear diffusion equation we use the control volume finite element method $[8,9,10]$ which is the well-known numerical method appropriate to the computation fluid dynamics. Also, we apply the Gauss-Newton method $[4,7]$ to find the minimum value of the quadratic functional which is defined in Section 2.

\section{Mathematical formulation}

In this section, we perform the mathematical formulation which represents the process of the wood drying on the basis of the equation (1.1)

$$
\frac{\partial m}{\partial t}=\frac{\partial}{\partial x}\left[D(m, t) \frac{\partial m}{\partial x}\right], \quad t>0, \quad 0<x<L
$$

where coefficient $D(m, t)$ is

$$
D(m, t)=p_{1} m^{p(t)}+A e^{-\mu\left(m-\nu_{0}\right)}, \quad p(t)=p_{2}+p_{3} e^{-p_{4} t} .
$$

Here $x \mathrm{~cm}$ indicates the location of the sample and the length of sample $L$ equals $9 \mathrm{~cm}$, and $t$ second indicates the time. In particular, $t=0$ or 1728000 which means the time the first or last data are obtained, are corresponding to 1st-day or 21st-day of measurement, respectively.

In order to solve the equation (1.1), we need one initial condition and two boundary conditions. The initial data is the type of

$$
m(x, 0)=m_{I}(x), \quad 0<x<L,
$$

where $m_{I}(x)$ is represented by the profile of moisture contents for the 1st day of measurement, and the boundary conditions are given by

$$
\begin{aligned}
& \frac{\partial m}{\partial x}(0, t)=0, \\
& -D(m(L, t), t) \frac{\partial m}{\partial x}(L, t)=B\left(m(L, t)-\nu_{0}\right), \quad t>0,
\end{aligned}
$$

Parameters $A, B$ and $\mu$ also can be determined, but we choose the values of the parameters $A=1.5 \mathrm{e}-9, B=1500$ and $\mu=150$, respectively. Also the minimum value $\nu_{0}=0.003929$ is attended for $x=L$. Hence $p_{1}, p_{2}, p_{3}$ and $p_{4}$ which are the coefficients concern with the moisture transfer are the parameter to be selected. 
For the purpose of selection of the parameters $P=\left[p_{1}, p_{2}, p_{3}, p_{4}\right]$, we define the minimization problem of the functional

$$
F(P)=\sum_{k=2}^{M} \int_{0}^{L}\left[m\left(x, t_{k}\right)-m_{e}\left(x, t_{k}\right)\right]^{2} d x,
$$

where $M=5, t_{k}$ 's are time moments corresponding to the 1st, 2nd, 7 th, 14th and the 21st day of measurement, $m_{e}\left(x, t_{k}\right)$ is profile of the experimental data for time $t_{k}$, and $m\left(x, t_{k}\right)$ is solution of the diffusion equation and conditions for the same time. Since we use $m\left(x, t_{1}\right)$ as the initial condition, we obtain $m\left(x, t_{1}\right)=m_{e}\left(x, t_{1}\right)$ immediately. So we start the summation with $k=2$ in the functional (2.6).

Our objective is selection the moisture transfer coefficient $D$ by comparing the experimental data $m_{e}(x, t)$ with numerically computed solution data $m(x, t)$. To obtain the solution data $m(x, t)$, we solve the nonlinear diffusion equation numerically by using control volume finite element method.

\section{Numerical Experiments}

In this section we introduce control volume finite element method (CVFEM) and Gauss-Newton method which are used to discretize the transport model equation and estimate the minimizers of the given functional, respectively. Also, we show the numerical results.

\subsection{Control Volume Finite Element Method}

We consider the initial-boundary value problem (2.1)-(2.5). In order to find the numerical solution of the problem by CVFEM, we introduce the uniform mesh

$$
\Phi=\left\{x_{i}=h i \mid 0=x_{0}<x_{1}<\cdots<x_{N}=L\right\},
$$

where $h=1 / N$ is spatial step size, $N$ is the number of nodes and construct an control volume corresponding to the nodes as shown in Figure 3.1.

Applying time discretization technique such as the backward Euler or the Crank-Nicolson schemes to (2.1), we have the following stationary equation at each time step

$$
\left(m-m^{(\text {prev })}\right) / \delta t-\frac{\partial}{\partial x}\left[D(m, t) \frac{\partial m}{\partial x}\right]=0,
$$




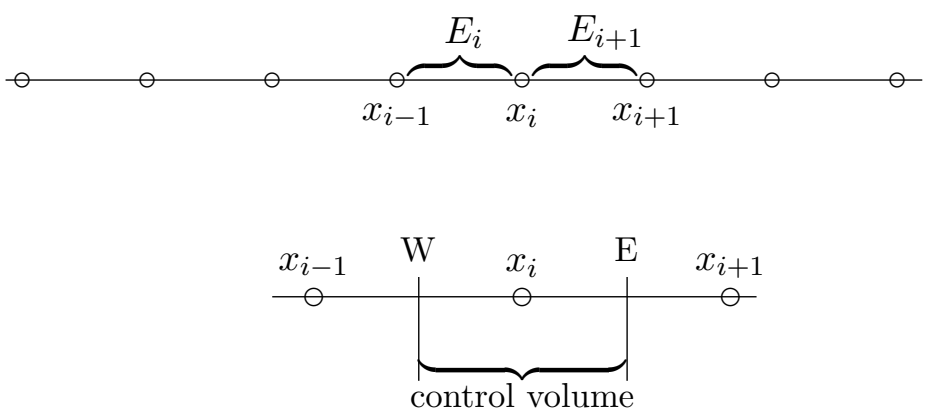

Figure 3.1. Construction of a one-dimensional finite elements and nodes(top) and control volumes(bottom).

where $m^{\text {(prev) }}$ means the value of the moisture contents at the previous time step and $\delta t$ means the time step size.

As shown in Figure 3.1, the computational domain is meshed with subinterval elements, and at each node $x_{i}$, the corresponding control volumes(CVs) are constructed. To obtain the discretized formulation of the stationary equation (3.2), we have integrating over the each $\mathrm{CV}$

$$
\frac{\operatorname{Area}(\mathrm{CV})}{\delta t}\left(m_{i}-m_{i}^{(\mathrm{prev})}\right)-\int_{\mathrm{CV}} \frac{\partial}{\partial x} \mathbf{J} d x=0,
$$

where $m_{i}$ is representative value of $m$ in the CV corresponding to $x_{i}$, i.e.,

$$
m_{i}=\frac{1}{\operatorname{Area}(\mathrm{CV})} \int_{\mathrm{CV}} m d x
$$

and the flux $\mathbf{J}=D(m, t) \frac{\partial m}{\partial x}$. By applying the Gauss divergence theorem, we have

$$
\alpha m_{i}-\sum_{f \in \mathfrak{F}_{\mathrm{CV}}}(\mathbf{J} \cdot \mathbf{n})_{f}=\alpha m_{i}^{(\text {prev })}
$$

where $\alpha=\frac{\operatorname{Area}(\mathrm{CV})}{\delta t}, \mathfrak{F}_{\mathrm{CV}}$ is the set of two end-points of the CV and $\mathbf{n}_{f}$ is outward unit normal vector. In order to evaluate the approximated value of the term $(\mathbf{J} \cdot \mathbf{n})_{f}$ at the end-point of the $\mathrm{CV}$, the value of $D(m, t)$ is given by an average value

$$
\begin{aligned}
\left.D(m, t)\right|_{\mathrm{W}} & =\frac{D\left(m_{i}, t\right)+D\left(m_{i-1}, t\right)}{2}, \text { and } \\
\left.D(m, t)\right|_{\mathrm{E}} & =\frac{D\left(m_{i}, t\right)+D\left(m_{i+1}, t\right)}{2},
\end{aligned}
$$


and the derivative is approximated numerically as

$$
\left.\frac{\partial m}{\partial x}\right|_{\mathrm{W}}=\frac{m_{i}-m_{i-1}}{x_{i}-x_{i-1}} \quad \text { and }\left.\quad \frac{\partial m}{\partial x}\right|_{\mathrm{E}}=\frac{m_{i+1}-m_{i}}{x_{i+1}-x_{i}} .
$$

Then we have the $N$ discrete analogue of the equations(3.2) as following:

$$
F_{i}(x):=
$$

(3.3) $\alpha\left(m_{i}^{(n+1)}-m_{i}^{(n)}\right)+\alpha_{i} m_{i}^{(n+1)}+\alpha_{i-1} m_{i-1}^{(n+1)}+\alpha_{i+1} m_{i+1}^{(n+1)}=0$,

where the superscript $(n+1)$ means the current time level $t^{(n+1)},(n)$ the previous time level $t^{(n)}$, and time step size is $\delta t=t^{(n+1)}-t^{(n)}$. However, the system of equations becomes nonlinear, and so iteration method like as Newton's method has been used.

\subsection{Nonlinear Least-Square Method and Gauss-Newton Method}

In order to determine the coefficients $P=\left[p_{1}, p_{2}, p_{3}, p_{4}\right]$ of diffusion equation, we define the objective functional to be minimized as follows:

$$
F(P)=\sum_{k=2}^{M} \sum_{i=0}^{N} \frac{1}{N}\left|m\left(x_{i}, t_{k}\right)-m_{e}\left(x_{i}, t_{k}\right)\right|^{2},
$$

The necessary condition to minimize the function at $P^{*}$ is

$$
\nabla F\left(P^{*}\right)=0 .
$$

Then the gradient of $F(P)$ is

$$
\nabla F(P)=\sum_{i=1}^{K} r_{i}(P) \nabla r_{i}(P)=J(P)^{T} r(P),
$$

where

$$
\begin{aligned}
r(P) & =\left(r_{1}, r_{2}, \cdots, r_{K}\right)^{T}, \\
r_{i}(P) & =\left|m\left(x_{i}, t_{k}\right)-m_{e}\left(x_{i}, t_{k}\right)\right|, \quad i=1, \cdots, K \\
K & =(N+1)(M-1)
\end{aligned}
$$


and the Jacobian matrix $J(P)$ of $r(P)$ is

$$
J(P)=\left(\begin{array}{cccc}
\frac{\partial r_{1}}{\partial p_{1}} & \frac{\partial r_{1}}{\partial p_{2}} & \frac{\partial r_{1}}{\partial p_{3}} & \frac{\partial r_{1}}{\partial p_{4}} \\
\frac{\partial r_{2}}{\partial p_{1}} & \frac{\partial r_{2}}{\partial p_{2}} & \frac{\partial r_{2}}{\partial p_{3}} & \frac{\partial r_{2}}{\partial p_{4}} \\
\vdots & \vdots & \vdots & \vdots \\
\frac{\partial r_{K}}{\partial p_{1}} & \frac{\partial r_{K}}{\partial p_{2}} & \frac{\partial r_{K}}{\partial p_{3}} & \frac{\partial r_{K}}{\partial p_{4}}
\end{array}\right) .
$$

However, this system of equations is nonlinear and difficult to compute. Hence the Newton's method is used to solve this system. The original Newton's iterative formula has the form.

$$
P^{(k+1)}=P^{(k)}-\left[J\left(P^{(k)}\right)^{T} J\left(P^{(k)}\right)+S\left(P^{(k)}\right)\right]^{-1} J\left(P^{(k)}\right)^{T} r\left(P^{(k)}\right),
$$

where Hessian $S(P)$ has the second-order derivative such as

$$
S(P)=\sum_{i=1}^{K} r_{i}(P) \nabla^{2} r_{i}(P) .
$$

However, second-order derivative term is expensive to compute and make the system ill-conditioned. Hence, by neglecting this second-order term in Newton's method, the simplified iteration is following:

$$
P^{(k+1)}=P^{(k)}-\left[J\left(P^{(k)}\right)^{T} J\left(P^{(k)}\right)\right]^{-1} J\left(P^{(k)}\right)^{T} r\left(P^{(k)}\right) .
$$

This equation is said to be the Gauss-Newton iteration. To make the iteration well-defined, it is required that the rank of the Jacobian matrix is 4. Obviously, the success of Gauss-Newton method will depend on the importance of the neglected second-order term $S(P)$. If $S\left(P^{*}\right)=$ 0 , the Gauss-Newton method would have quadratic convergence rate. In general, the Lebenverg-Marquardt method is used to improve the numerical stability and convergence. These parameters were adjusted by the fitting procedure until the best agreement between experimental and simulation was obtained.

\subsection{Numerical Results}

As explained above, we find the numerical solution by solving the transport equations in terms of control volume finite element methods also we find the value of the parameters $P=\left[p_{1}, p_{2}, p_{3}, p_{4}\right]$.

In order to minimize the functional (3.4) successfully by Newton method, choosing the initial values of parameters is very important. In this paper, initial values $P=\left[p_{1}, p_{2}, p_{3}, p_{4}\right]$ are selected to $P=$ 
[0.0006, 4, 2, 0.000003]. These initial values of parameters are improved by the Gauss-Newton method. For the purpose of it, we solve numerically the problem (2.1)-(2.5) for this values of parameters by using the discretized CVFEM (3.3). Figure 3.2 shows the numerical solution of the moisture concentration for initial values of parameters. The value of the functional (3.4) is $F(P)=0.00101134$. As shown in Figure 3.2, the coincidence between two data is poor. These values of parameters are improved by Gauss-Newton method as explained in Section 3.2. The process of improvement is demonstrated in Table 3.1, and then we get the result values of the parameters $P=[0.0004759109$, $3.540119,1.666714,0.000002939047]$ after 7 -iterations. For the improved values of parameters, we have the good coincidence between two data as shown in Figure 3.3 and the value of functional is decreased to $F(P)=0.0000171405$. Also, we can solve the minimization problem for the 7-parameters $P=\left[p_{1}, p_{2}, p_{3}, p_{4}, A, B, \mu\right]$ by adding the 3 -parameters $A, B$ and $\mu$. Then we have similar result values of the parameters $P=$ $[0.00046269,3.7330,1.775,0.000002918,0.000000001562,1594.6,123.3]$ and the value of functional $F(P)=0.0000154723$ after 7 -iterations.

\begin{tabular}{|c|c|c|c|c|c|}
\hline $\mathrm{n}$ & $P_{0}$ & $P_{1}$ & $P_{2}$ & $P_{3}$ & $\mathrm{~F}(\mathrm{P})$ \\
\hline 0 & $6.000000 \mathrm{e}-4$ & 4.000000 & 2.000000 & $3.000000 \mathrm{e}-6$ & 0.001011340507 \\
\hline 1 & $4.802076 \mathrm{e}-4$ & 3.413793 & 1.203327 & $2.198375 \mathrm{e}-6$ & 0.000430344776 \\
\hline 2 & $3.947006 \mathrm{e}-4$ & 3.730629 & 2.080626 & $4.131628 \mathrm{e}-6$ & 0.000310508770 \\
\hline 3 & $3.824236 \mathrm{e}-4$ & 3.433472 & 1.173006 & $2.339742 \mathrm{e}-6$ & 0.000259365691 \\
\hline 4 & $3.425655 \mathrm{e}-4$ & 3.519996 & 2.078242 & $3.887038 \mathrm{e}-6$ & 0.000130385044 \\
\hline 5 & $4.563707 \mathrm{e}-4$ & 3.549425 & 1.394136 & $2.740464 \mathrm{e}-6$ & 0.000065441444 \\
\hline 6 & $4.762567 \mathrm{e}-4$ & 3.535573 & 1.724402 & $3.024057 \mathrm{e}-6$ & 0.000018110429 \\
\hline 7 & $4.759109 \mathrm{e}-4$ & 3.540119 & 1.666714 & $2.939047 \mathrm{e}-6$ & 0.000017140492 \\
\hline
\end{tabular}

TABLE 3.1. The values of parameters after $n$-th iteration by Gauss-Newton method 


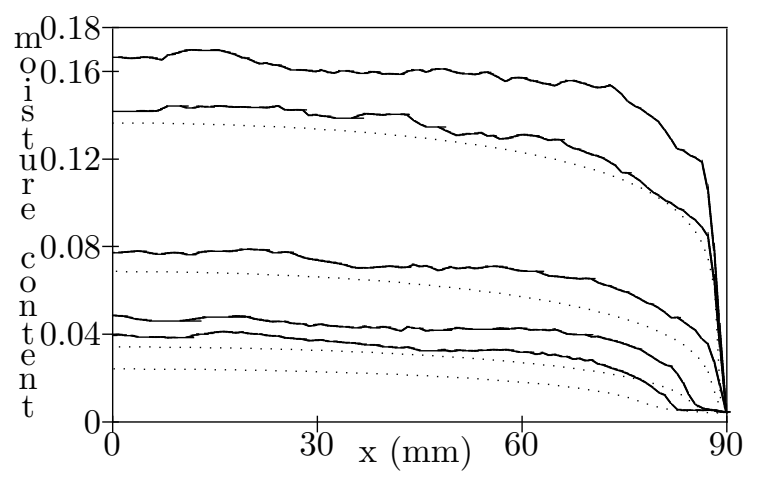

Figure 3.2. Computational(dashed lines) versus experimental(solid lines) profile of the moisture concentration for initial values of parameters.

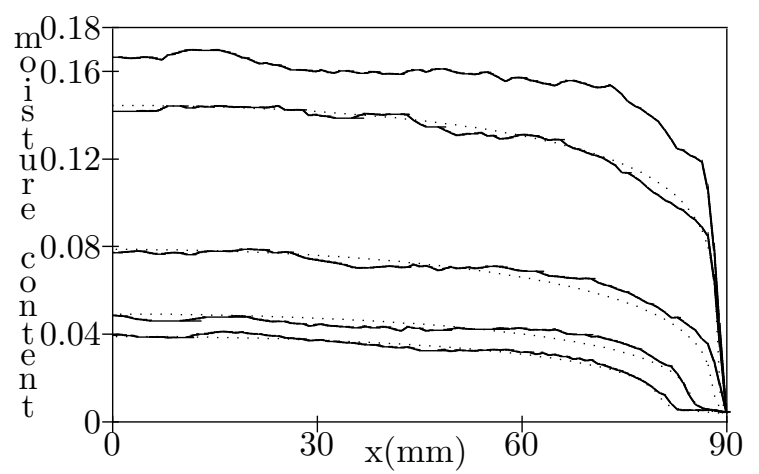

Figure 3.3. Computational(dashed lines) versus experimental(solid lines) profile of the moisture concentration for improved values of parameters by Newton method.

\section{Concluding Remarks}

On the basis of the control volume finite element method of solving the nonlinear diffusion equation with initial and boundary conditions, by means of solution of direct problem, a method of searching of the moisture transfer coefficient function $D(m, t)$ is presented for the first time in the form of sum of power and the exponential function which depends on the moisture concentration $m$. The exponent of the power function represents a time dependent function $p(t)$. The values of the parameters is determined by the functional minimization regarding to 
these parameters. A complete CVFE method is suitable for resolving nonlinear transport equations on one-dimensional meshes.

We have received the good coincidence of calculated and experimental profiles of moisture concentration for values of parameters

$P=\left[p_{1}, p_{2}, p_{3}, p_{4}\right]=[0.0004759109,3.540119,1.666714,0.000002939047]$, and for the diffusion transfer coefficient

$$
D(m, t)=0.000476 m^{p(t)}+1.5 \times 10^{-9} e^{-150(m-0.003929)},
$$

where $p(t)=3.54+1.67 e^{-2.93 \times 10^{-6} t}$.

\section{References}

[1] I. V. Amirkhanov, E. Pavlusova, M. Pavlus, T. P. Puzynina, I. V. Puzynin and I. Sarhadov, Numerical solution of an inverse diffusion problem for the moisture transfer coefficient in a porous material, Materials anf Structures 41 (2008), 335-344.

[2] Baliga and S. V. Patankar, A New Finite Element Formulation for ConvectionDiffusion Problems, Numerical Heat Transfer 3 (1980), 393-409.

[3] W. J. Ferguson, A Control Volume Finite Element Numerical Simulation of the high temperature drying of Spruce, Drying Technology 13 (1995), 607-634.

[4] W. Kang, Y. H. Lee, W, Y. Chung and H. R. Xu, Parameter estimation of moisture diffusivity in wood by an inverse method, Journal of Wood Science $\mathbf{5 5}$ (2009), 83-90.

[5] S. V. Patankar, Numerical heat transfer and fluid flow, Hemisphere Publ. Corp., New York, 1980

[6] H. Pleinert, H. Sadouki, F. H. Wittmann, Determination of moisture distribution in porous building materials by neutron transmission analysis, Mater. Struct. 31 (1998), 218-224.

[7] Wenyu Sun and Ya-Xiang Yuan, Optimization Theory and Methods Nonlinear Programming, Springer, (2006)

[8] S. L. Truscott and I. W. Turner, Heterogeneous three-dimensional computational model for wood drying, Applied Mathematical Modelling 29 (2005), 381-410.

[9] H. K. Versteeg and W. Malalasekera, An Introduction to Computational Fluid Dynamics The Finite Volume Method, Pearson Education Limited (2007).

[10] V. R. Voller, Basic Control Volume Finite Element Methods for Fluids and Solids IISc Research Monographs Series Vol. 1, World Scientific Publishing Co. (2009).

Yong Hun Lee

Department of Mathematics

(Institute of Pure and Applied Mathematics),

Chonbuk National University,

Chonbuk, 561-756, Republic of Korea

E-mail: 1yh229@chonbuk.ac.kr 